\title{
The association between XRCCI Arg399GIn polymorphism and risk of leukemia in different populations: a meta-analysis of case-control studies
}

\author{
Fang Wang ${ }^{1,2, *}$ \\ Qian Zhao ${ }^{1,2, *}$ \\ Hai-rong $\mathrm{He}^{3}$ \\ Ya-jing Zhai ${ }^{4}$ \\ Jun $\mathrm{Lu}^{3}$ \\ Hai-bo $\mathrm{Hu}^{\prime}$ \\ Jin-song Zhou' \\ Yong-hua Yang ${ }^{5}$ \\ Yuan-jie $\mathrm{Li}^{\prime}$

\begin{abstract}
'Department of Human Anatomy, Histology and Embryology, School of Basic Medical Sciences, Xi'an Jiaotong University Health Science Centre, ${ }^{2}$ College of Pharmacy, Xi'an Medical University, ${ }^{3}$ Clinical Research Center, ${ }^{4}$ Department of Pharmacy, ${ }^{5}$ Department of Pediatrics, The First Affiliated Hospital, Xi'an Jiaotong University, Xi'an, People's Republic of China
\end{abstract}

*These authors contributed equally to this work

This article was published in the following Dove Press journal:

OncoTargets and Therapy

6 November 2015

Number of times this article has been viewed

Background: Associations between Arg399Gln single-nucleotide polymorphism (SNP) in the $X R C C 1$ gene and leukemia susceptibility have been studied extensively, however, the results are inconsistent. The aim of this study was to determine these associations using metaanalytical methods.

Methods: A meta-analysis was performed to examine the associations between XRCC1 Arg399Gln SNP and leukemia risk. A literature search of PubMed and Web of Science databases was conducted to identify relevant studies published up to March 10, 2015. The references of the retrieved articles were also screened. All the statistical analyses were conducted using Review Manager software.

Results: The XRCC1 Arg399Gln SNP was found to be associated with increased childhood risk of acute lymphoblastic leukemia among Asians under the dominant (odds ratio [OR] 2.11, 95\% confidence interval [CI] 1.50-2.97, $P<0.0001$ ), allele contrast (OR 1.72, 95\% CI 1.33-2.23, $P<0.0001$ ), and homozygote contrast (OR 2.34, 95\% CI 1.25-4.36, $P=0.008$ ) models. However, no association was found in Caucasians between the SNP and risk of either chronic myeloid leukemia or chronic lymphocytic leukemia under any contrast model.

Conclusion: The findings of the current meta-analysis indicate that the XRCC1 Arg399Gln SNP is a risk factor for childhood lymphoblastic leukemia in Asians.

Keywords: Arg399Gln, AML, ALL, CML, CLL, susceptibility

\section{Introduction}

Leukemia is a malignant neoplasm of blood-forming tissues ${ }^{1}$ that can be classified into the following four groups according to the cell type and growth rate: acute myeloid leukemia (AML), acute lymphocytic leukemia (ALL), chronic myeloid leukemia (CML), and chronic lymphocytic leukemia (CLL). ${ }^{2}$ Despite numerous studies on leukemogenesis, the mechanism underlying the development of these cancers is yet to be fully elucidated.

DNA repair pathways play a vital role in maintaining genetic integrity, and it is becoming clear that defects in repair pathways are connected to many different types of diseases, including leukemia. ${ }^{3}$ It has been reported that impaired DNA repair may be associated with increased susceptibility to human cancers. ${ }^{4}$ The $X R C C 1$ gene is one of the most important DNA repair genes, and plays a key role in the process of base excision repair. ${ }^{1}$ XRCC1 single-nucleotide polymorphism (SNP) Arg399Gln at codon 399 has been extensively examined and is associated with diminished capacity to remove DNA adducts, causing DNA damage due to oxidation. ${ }^{5}$ Therefore, the Arg399Gln SNP may contribute to leukemia.

Correspondence: Yuan-jie Li Department of Human Anatomy, Histology and Embryology, School of Basic Medical Sciences, Xi'an Jiaotong University Health Science Centre, 76 West Yanta Road, Xi'an 71006I, People's Republic of China

Tel +8629853236l4

Fax +862985323473

Email liyuanjie_xjtu@I63.com 
Studies of the association between XRCC1 SNPs and leukemia have produced conflicting findings, possible reasons for which include differences in ethnicity and sample size. The aim of the present meta-analysis was to obtain a more accurate picture with regard to the role of the XRCC1 Arg399Gln SNP in the risk of leukemia.

\section{Materials and methods}

\section{Search strategy}

Computerized searches of the PubMed and Web of Science databases were conducted using a combination of the following keywords: "XRCC1" ("X-ray repair cross complementing 1" OR "X-ray repair cross complementing group 1" OR "X-ray cross complementing repair gene 1"), "AML" ("acute myeloid leukemia" OR "acute myeloblastic leukemia" OR "acute myelocytic leukemia"), "ALL" ("acute lymphocytic leukemia" OR "acute lymphoblastic leukemia"), "CLL" ("chronic lymphocytic leukemia" OR "chronic lymphoblastic leukemia"), "CML" (“chronic myeloid leukemia" OR "chronic myelocytic leukemia" OR "chronic myeloblastic leukemia"), "leukemia", and "hematological malignancies". All references cited in the selected studies were also reviewed to identify additional relevant work.

\section{Inclusion criteria}

Published studies were included if they satisfied the following criteria:

1. Only studies published in journals in English were included in the analysis;

2. Used a case-control design;

3. Focused on the association between the XRCC1 Arg399Gln SNP and risk of AML, ALL, CML, or CLL;

4. Provided sufficient data on the distribution of the XRCC1 Arg399Gln SNP in leukemia and in controls, or sufficient information for such data to be calculated.

\section{Data extraction}

The following information was extracted from each study: first author, publication year, subjects' ethnicity, disease type, number of cases and controls, Hardy-Weinberg equilibrium (HWE) for the controls' distribution of genotypes, and main results about associations between the XRCC1 Arg399Gln SNP and risk of leukemia.

\section{Statistical analysis}

The association between the XRCC1 Arg399Gln SNP and risk of different types of leukemia in different populations was evaluated under the allele contrast (Gln versus Arg), homozygote contrast (Gln/Gln versus Arg/Arg), dominant ( $\mathrm{Gln} / \mathrm{Gln}+\mathrm{Arg} / \mathrm{Gln}$ versus Arg/Arg), and recessive (Gln/ Gln versus $\mathrm{Arg} / \mathrm{Gln}+\mathrm{Arg} / \mathrm{Arg}$ ) models. Studies wherein the distribution of the XRCC1 Arg399Gln genotypes among the controls deviated from HWE were excluded from the meta-analysis.

Review Manager software (version 5.3) was used for the meta-analysis. Raw data of genotype distribution were used to calculate the study-specific estimates of odds ratios (ORs) and 95\% confidence intervals (CIs). The heterogeneity of the studies was assessed using Cochran's $Q$ test, and was considered statistically significant at $P<0.10$. Heterogeneity was also quantified using the heterogeneity index $\left(I^{2}\right)$ statistic, with $R^{2}>50 \%$ indicating the presence of a high degree of heterogeneity. ${ }^{6}$

The strength of associations between the XRCC1 Arg399Gln SNP and different types of leukemia risk was assessed by the ORs and the corresponding 95\% CIs. The significance of the pooled ORs was determined by the $Z$-test, and the threshold for significance was set at $P<0.05$. The fixed-effects model (Mantel-Haenszel methods) was used when there was no substantial heterogeneity. In the event of substantial heterogeneity, sensitivity analysis was performed by excluding individual studies; outlying studies were identified and excluded, and the $I^{2}$ estimates for these different sets of studies were examined. The random-effects model (DerSimonian and Laird's method) ${ }^{7}$ was used when removal of particular studies did not render the heterogeneity insignificant (ie, $I^{2}<50 \%$ ).

Potential publication bias was estimated by constructing funnel plots. If most of the data appeared at the top of a funnel plot and was distributed roughly symmetrically, this would suggest the absence of obvious publication bias, and vice versa. ${ }^{8}$ There was no need to construct funnel plots when there were too few (ie, less than five) analyzed studies.

\section{Results}

\section{Overview of the study characteristics}

A flowchart depicting the study selection process is shown in Figure 1. In total, 746 articles were selected on the basis of various combinations of the keywords listed in the "Methods and materials" section. Checking for duplicates resulted in the removal of 660 articles. Of the remaining 86 articles, 65 were excluded for the following reasons: lack of relevance, since the articles did not explore the association between $X R C C 1$ SNPs and leukemia risk ( $n=23)$; did not focus on susceptibility to leukemia $(n=24)$; 


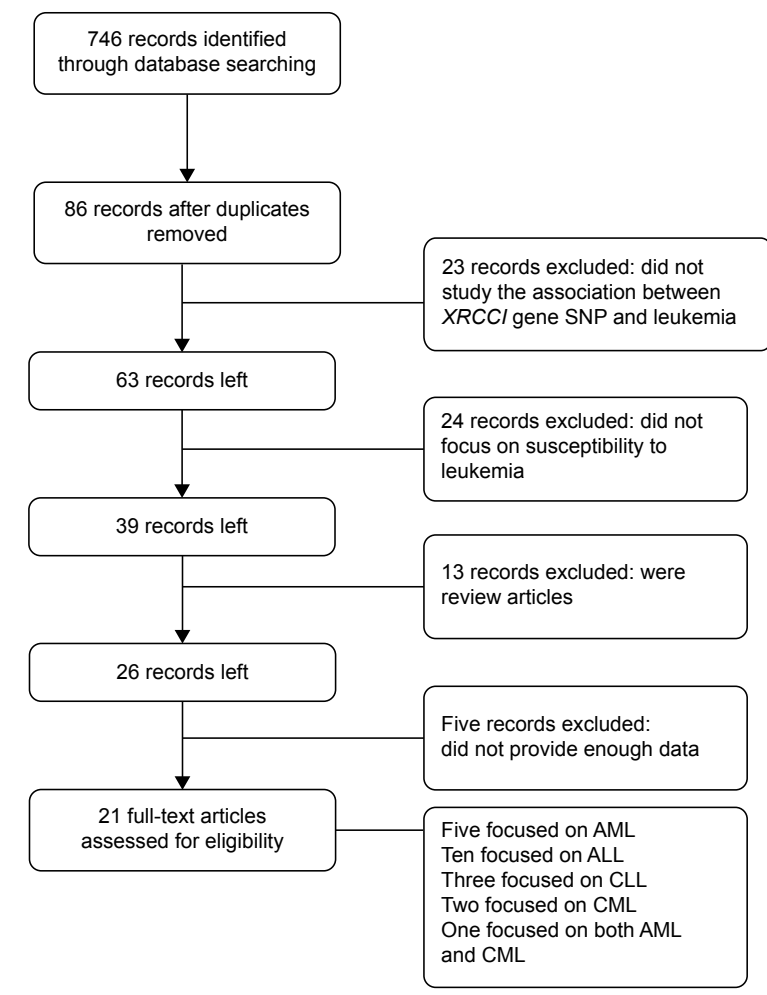

Figure I Flowchart of study selection.

Abbreviations: ALL, acute lymphocytic leukemia; AML, acute myeloid leukemia; CLL, chronic lymphocytic leukemia; CML, chronic myeloid leukemia; SNP, singlenucleotide polymorphism. were review articles $(n=13)$; or provided insufficient data $(n=5)$. Thus, only 21 articles qualified for inclusion in this meta-analysis, among which $5,10,3,2$, and 1 focused on AML, ${ }^{3,9-12}$ ALL, ${ }^{13-22}$ CLL, ${ }^{23-25} \mathrm{CML},{ }^{26,27}$ and both AML and $\mathrm{CML},{ }^{28}$ respectively.

Basic data for every eligible study were extracted and are listed in Table 1. In one article, ${ }^{16}$ in which different ethnic populations were evaluated, data for each ethnicity were collected separately and were treated in the present analysis as independent studies. Moreover, another included article ${ }^{28}$ provided separate data for two types of leukemia; further, data for each leukemia type were analyzed as if they were from different studies. As a result, data from a final total of 23 studies were finally included in this meta-analysis. However, the genetic distributions of the control groups did not conform with HWE in three of these studies, ${ }^{10,11,27}$ which were thus excluded from the meta-analysis. The distribution of the remaining studies regarding meta-analysis of the association between XRCC1 Arg399Gln SNP and risk of each type of leukemia was as follows: ALL, eleven studies $(1,088$ cases and 1,588 controls); AML, four studies ( 345 cases and 651 controls); CML, two studies (338 cases and 406 controls); and CLL, three studies (712 cases and 614 controls).

Table I Study characteristics

\begin{tabular}{|c|c|c|c|c|c|c|}
\hline Study & Year & Ethnicity & Cancer & Case/control & $P_{\text {HWE }}$ in control & Main results \\
\hline Abramenko et al ${ }^{24}$ & 2012 & Caucasian & CLL & $178 / 103$ & 0.4845 & $N$ \\
\hline Annamaneni et $\mathrm{al}^{27}$ & 2013 & Asian & CML & $350 / 350$ & 0.0000 & $(+)$ \\
\hline Banescu et $\mathrm{al}^{9}$ & 2014 & Caucasian & AML & $69 / 147$ & 0.2195 & $(+)$ \\
\hline Banescu et $\mathrm{al}^{26}$ & 2014 & Caucasian & CML & $156 / 180$ & 0.8041 & $\mathrm{~N}$ \\
\hline Batar et al ${ }^{19}$ & 2009 & Caucasian & ALL & $70 / 75$ & 0.9687 & $\mathrm{~N}$ \\
\hline Canalle et $\mathrm{al}^{16}$ & 2011 & Caucasian & ALL & $178 / 223$ & 0.3209 & $\mathrm{~N}$ \\
\hline Canalle et $\mathrm{al}^{16}$ & 2011 & Mixed & ALL & $28 /|4|$ & 0.6087 & $\mathrm{~N}$ \\
\hline Celkan et $\mathrm{al}^{20}$ & 2008 & Caucasian & ALL & $52 / 60$ & 0.8248 & $\mathrm{~N}$ \\
\hline Deligezer et $\mathrm{a}^{28}$ & 2007 & Caucasian & AML & $72 / 226$ & 0.7629 & $\mathrm{~N}$ \\
\hline Deligezer et $\mathrm{a}^{28}$ & 2007 & Caucasian & CML & $182 / 226$ & 0.7629 & $\mathrm{~N}$ \\
\hline Dincer et $\mathrm{al}^{14}$ & 2015 & Caucasian & ALL & $30 / 30$ & 0.1259 & $\mathrm{~N}$ \\
\hline Duman et $\mathrm{a}^{23}$ & 2012 & Caucasian & CLL & $73 / 50$ & 0.3636 & $(+)$ \\
\hline Ganster et $\mathrm{al}^{25}$ & 2009 & Caucasian & CLL & $46 I / 46 I$ & 0.8988 & $\mathrm{~N}$ \\
\hline Goricar et $\mathrm{al}^{13}$ & 2015 & Caucasian & ALL & $121 / 184$ & NA & $\mathrm{N}$ \\
\hline Joseph et $\mathrm{al}^{22}$ & 2005 & Asian & ALL & $117 / 117$ & 0.0619 & $(+)$ \\
\hline Kim et al"I & 2012 & Asian & AML & $415 / 1,700$ & 0.0058 & $\mathrm{~N}$ \\
\hline Meza-Espinoza et al ${ }^{18}$ & 2009 & Mixed & ALL & $120 / 120$ & 0.8992 & $N$ \\
\hline Ozcan et al ${ }^{12}$ & 2011 & Caucasian & AML & $36 / 100$ & 0.4692 & $(-)$ \\
\hline Pakakasama et $\mathrm{al}^{21}$ & 2007 & Asian & ALL & $108 / 317$ & 0.5146 & $(+)$ \\
\hline Seedhouse et al ${ }^{3}$ & 2002 & Caucasian & AML & $168 / 178$ & $0.054 I$ & $(-)$ \\
\hline Sorour et $\mathrm{al}^{10}$ & 2013 & Caucasian & AML & $90 / 60$ & 0.0245 & $(+)$ \\
\hline Stanczyk et al ${ }^{15}$ & 2011 & Caucasian & ALL & $97 / \mid 31$ & 0.2815 & $\mathrm{~N}$ \\
\hline Tumer et al ${ }^{17}$ & 2010 & Caucasian & ALL & $167 / 190$ & 0.5694 & $(+)$ \\
\hline
\end{tabular}

Abbreviations: N, no association between SNP and leukemia susceptibility; (+), SNP associated with increased leukemia susceptibility; (-), SNP associated with decreased leukemia susceptibility; NA, not available; SNP, single-nucleotide polymorphism; CLL, chronic lymphocytic leukemia; CML, chronic myeloid leukemia; AML, acute myeloid leukemia; ALL, acute lymphocytic leukemia; $\mathrm{P}_{\text {HWE }}$ in control, probability of adherence to the Hardy-Weinberg equilibrium. 


\section{Meta-analysis results}

Table 2 lists the main results of the meta-analysis. The findings are discussed for each leukemia type separately in the following section.

\section{AML}

A meta-analysis of the four studies that focused on AML was performed on data from 345 cases and 651 controls. The subjects in all four studies were Caucasians. A high degree of heterogeneity was observed under the recessive $\left(I^{2}=79 \%, P=0.003\right)$, dominant $\left(I^{2}=70 \%, P=0.02\right)$, allele contrast $\left(I^{2}=84 \%, P=0.0003\right)$, and homozygote contrast $\left(I^{2}=82 \%\right.$, $P=0.0007)$ models. Therefore, random-effects modeling was performed for the four contrast models. The meta-analysis for these studies revealed no association between the XRCC1 Arg399GIn SNP and the risk of AML under the recessive model (OR 0.89, 95\% CI 0.33-2.44, $P=0.83$ ), dominant (OR $0.97,95 \%$ CI $0.58-1.63, P=0.91$ ), allele contrast (OR 0.94, 95\% CI $0.55-1.59, P=0.81$ ), or homozygote contrast models (OR 0.86, 95\% CI 0.27-2.78, $P=0.81$ ).

In view of the high degree of heterogeneity, a sensitivity analysis was conducted, and the study by Banescu et $\mathrm{al}^{9}$ was found to be an outlier. Removal of their data from the analysis reduced the degree of heterogeneity under the recessive and dominant models, but not sufficiently so under the allele contrast $\left(I^{2}=65 \%, P=0.06\right.$; Figure $\left.2 \mathrm{~A}\right)$ and homozygote contrast models $\left(I^{2}=59 \%, P=0.09\right.$; Figure $\left.2 \mathrm{~B}\right)$. Fixed-effects modeling was thus performed for the recessive and dominant models, and finally, the SNP was associated with AML risk under the recessive model (OR 0.58, 95\% CI $0.38-0.89, P=0.01$; Figure 2 C) but not under the dominant model (OR 0.79, 95\% CI 0.58-1.07, $P=0.13$; Figure 2D). Therefore, the result for AML under the recessive model is sensitive to the study by Banescu et al. ${ }^{9}$

\section{ALL}

Eleven of the 23 included studies investigated the distribution of XRCC1 SNP in childhood ALL cases and controls and provided sufficient data for analysis. A meta-analysis of these eleven studies was performed, and included data from 1,088 cases and 1,588 controls.

The forest plot revealed no heterogeneity under the recessive $\left(P=0.65, I^{2}=0 \%\right)$ and homozygote contrast $(P=0.50$, $\left.I^{2}=0 \%\right)$ models, and $I^{2}<50 \%$ under the allele contrast $\left(P=0.05, I^{2}=46 \%\right)$ model. The fixed-effects model was therefore used for these three contrast models. The degree

Table 2 Odds ratios (ORs) and heterogeneity results for XRCCI Arg399Gln meta-analysis

\begin{tabular}{|c|c|c|c|c|c|c|c|}
\hline \multirow[t]{2}{*}{ Type of cancer } & \multirow[t]{2}{*}{ Studies (n) } & \multirow[t]{2}{*}{ Ethnicity } & \multirow[t]{2}{*}{ Contrast models } & \multicolumn{2}{|l|}{ ORs (95\% Cls) } & \multirow[t]{2}{*}{$I^{2}$} & \multirow[t]{2}{*}{$P$-value } \\
\hline & & & & Fixed-effects model & Random-effects model & & \\
\hline \multirow[t]{12}{*}{ ALL } & 7 & Caucasian & Recessive model & I. $10(0.79,1.53)$ & & $0 \%$ & 0.57 \\
\hline & & & Dominant model & $1.00(0.82,1.22)$ & & $34 \%$ & 0.99 \\
\hline & & & Allele contrast & $1.07(0.91,1.26)$ & & $8 \%$ & 0.39 \\
\hline & & & Homozygote contrast & $1.18(0.82,1.69)$ & & $0 \%$ & 0.37 \\
\hline & 2 & Asian & Recessive model & $1.70(0.94,3.08)$ & & $0 \%$ & 0.08 \\
\hline & & & Dominant model & $2.11(1.50,2.97)$ & & $0 \%$ & $<0.000$ I \\
\hline & & & Allele contrast & $1.72(1.33,2.23)$ & & $0 \%$ & $<0.000$ I \\
\hline & & & Homozygote contrast & $2.34(1.25,4.36)$ & & $0 \%$ & 0.008 \\
\hline & 2 & Mixed & Recessive model & $\mathrm{I} .60(0.74,3.48)$ & & $0 \%$ & 0.23 \\
\hline & & & Dominant model & & $0.88(0.36,2.16)$ & $70 \%$ & 0.78 \\
\hline & & & Allele contrast & I.II $(0.79,1.55)$ & & $47 \%$ & 0.56 \\
\hline & & & Homozygote contrast & I.56 (0.7I, 3.42) & & $0 \%$ & 0.27 \\
\hline \multirow[t]{4}{*}{ AML } & 4 & Caucasian & Recessive model & $0.58(0.38,0.89)$ & & $44 \%$ & 0.01 \\
\hline & & & Dominant model & $0.79(0.58,1.07)$ & & $49 \%$ & 0.13 \\
\hline & & & Allele contrast & & $0.75(0.50,1.13)$ & $65 \%$ & 0.17 \\
\hline & & & Homozygote contrast & & $0.56(0.22, I .43)$ & $59 \%$ & 0.22 \\
\hline \multirow[t]{4}{*}{ CLL } & 3 & Caucasian & Recessive model & I.II $(0.79,1.57)$ & & $49 \%$ & 0.54 \\
\hline & & & Dominant model & $1.09(0.86,1.39)$ & & $0 \%$ & 0.46 \\
\hline & & & Allele contrast & $1.08(0.91,1.28)$ & & $0 \%$ & 0.40 \\
\hline & & & Homozygote contrast & $1.16(0.80,1.68)$ & & $32 \%$ & 0.44 \\
\hline \multirow[t]{4}{*}{ CML } & 2 & Caucasian & Recessive model & $0.99(0.62,1.57)$ & & $0 \%$ & 0.97 \\
\hline & & & Dominant model & $1.11(0.83,1.49)$ & & $0 \%$ & 0.46 \\
\hline & & & Allele contrast & $1.06(0.85,1.32)$ & & $0 \%$ & 0.60 \\
\hline & & & Homozygote contrast & I.05 $(0.65,1.7 \mathrm{I})$ & & $0 \%$ & 0.84 \\
\hline
\end{tabular}

Abbreviations: Cls, confidence intervals; CLL, chronic lymphocytic leukemia; CML, chronic myeloid leukemia; AML, acute myeloid leukemia; ALL, acute lymphocytic leukemia. 


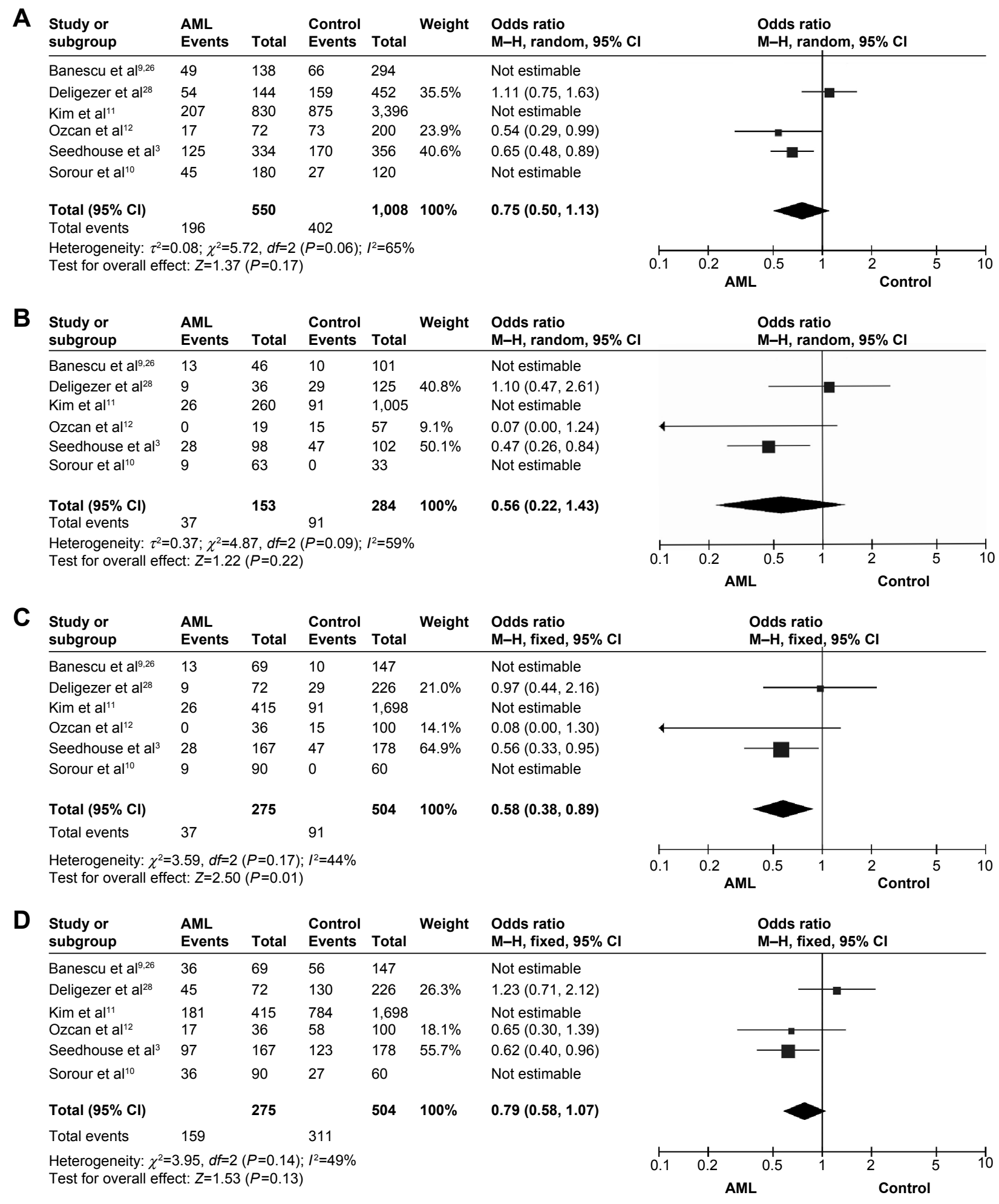

Figure 2 Meta-analysis of the association between XRCCI Arg399GIn SNP and AML risk under the allele contrast model (A), the homozygote contrast model (B), the recessive model (C), and the dominant model (D).

Abbreviations: SNP, single-nucleotide polymorphism; AML, acute myeloid leukemia; $\mathrm{Cl}$, confidence interval; M-H, Mantel-Haenszel.

of heterogeneity was high $\left(I^{2}=62 \%, P=0.003\right)$ under the dominant model; therefore, a random-effects model was employed under that model. Finally, an association was found between the SNP and increased childhood ALL risk under the allele contrast (OR 1.21, 95\% CI 1.06-1.37, $P=0.003$ ) and homozygote contrast (OR 1.41, 95\% CI 1.06-1.88, $P=0.02)$ models. The funnel plot did not reveal any obvious publication bias (Figure 3). 

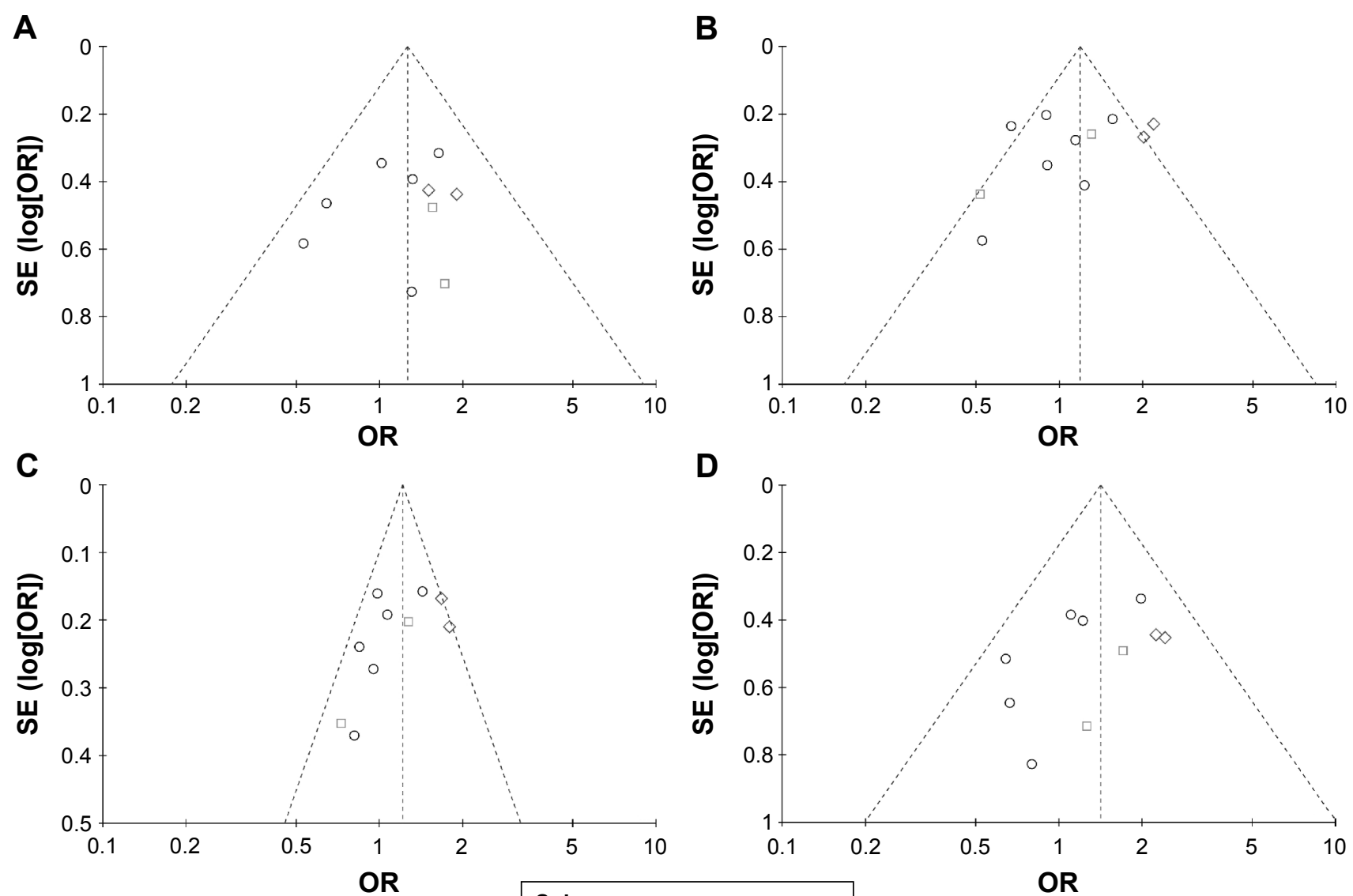

Subgroups

o Caucasian $\diamond$ Asian $\sqsubset$ Mixed

Figure 3 The funnel plots of meta-analysis for the association between XRCCI Arg399GIn SNP and ALL risk under the recessive model (A), the dominant model (B), the allele contrast model (C), and the homozygote contrast model (D).

Abbreviations: SNP, single-nucleotide polymorphism; ALL, acute lymphocytic leukemia; SE, standard error of the mean; OR, odds ratio.

As the different results obtained among the included studies could be attributable to population differences in Arg399Gln mutation frequency or linkage disequilibrium block, subgroup analysis according to race was conducted. In the Caucasian and Asian subgroups, heterogeneity was not found to be large $\left(I^{2}<50 \%\right)$ under any contrast model. Therefore, the fixed-effects model was used in Caucasian and Asian subgroups under any contrast model. The Arg399Gln SNP was then found to significantly increase the risk of childhood ALL only among Asians, under the dominant (OR 2.11, 95\% CI 1.50-2.97, $P<0.0001$; Figure 4B), allele contrast (OR 1.72, 95\% CI 1.33-2.23, $P<0.0001$; Figure 4C), and homozygote contrast (OR 2.34, 95\% CI 1.25-4.36, $P=0.008$; Figure 4D) models. No association was observed under the recessive model (OR 1.70, 95\% CI 0.94-3.08, $P=0.08$; Figure 4A). Furthermore, no association between the XRCC1 Arg399Gln SNP and risk of childhood ALL was found under any contrast model among Caucasian or mixed-race populations (Figure 4).
CML

CML was surveyed in two studies, involving data from 338 cases and 406 controls, all of whom were Caucasian. No heterogeneity was found between the two included studies under any contrast model, and so the fixed-effects model was used. Overall, the meta-analysis for those studies revealed no association between the XRCC1 Arg399Gln SNP and risk of CML under any contrast model (Table 2).

\section{CLL}

The association between the XRCC1 Arg399Gln SNP and risk of CLL was researched in three studies with 712 cases and 614 controls, all of whom were Caucasian. The forest plot revealed a high degree of heterogeneity. Random-effects modeling was thus performed for the four contrast models. The meta-analysis for these studies revealed no association between the SNP and risk of CLL under any contrast model.

In view of such a high degree of heterogeneity, a sensitivity analysis was conducted, revealing the study of Duman et a ${ }^{23}$ 
A

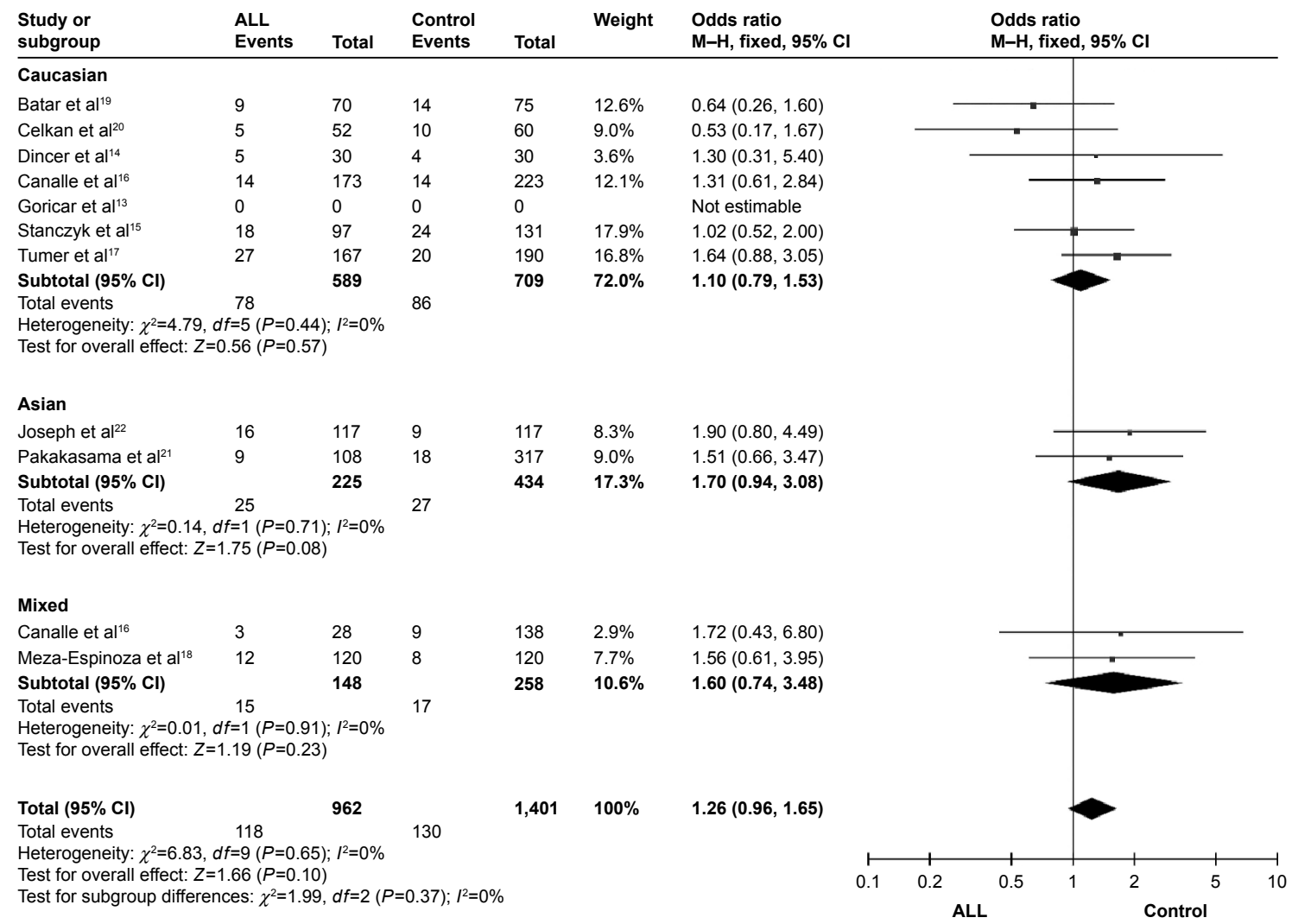

B

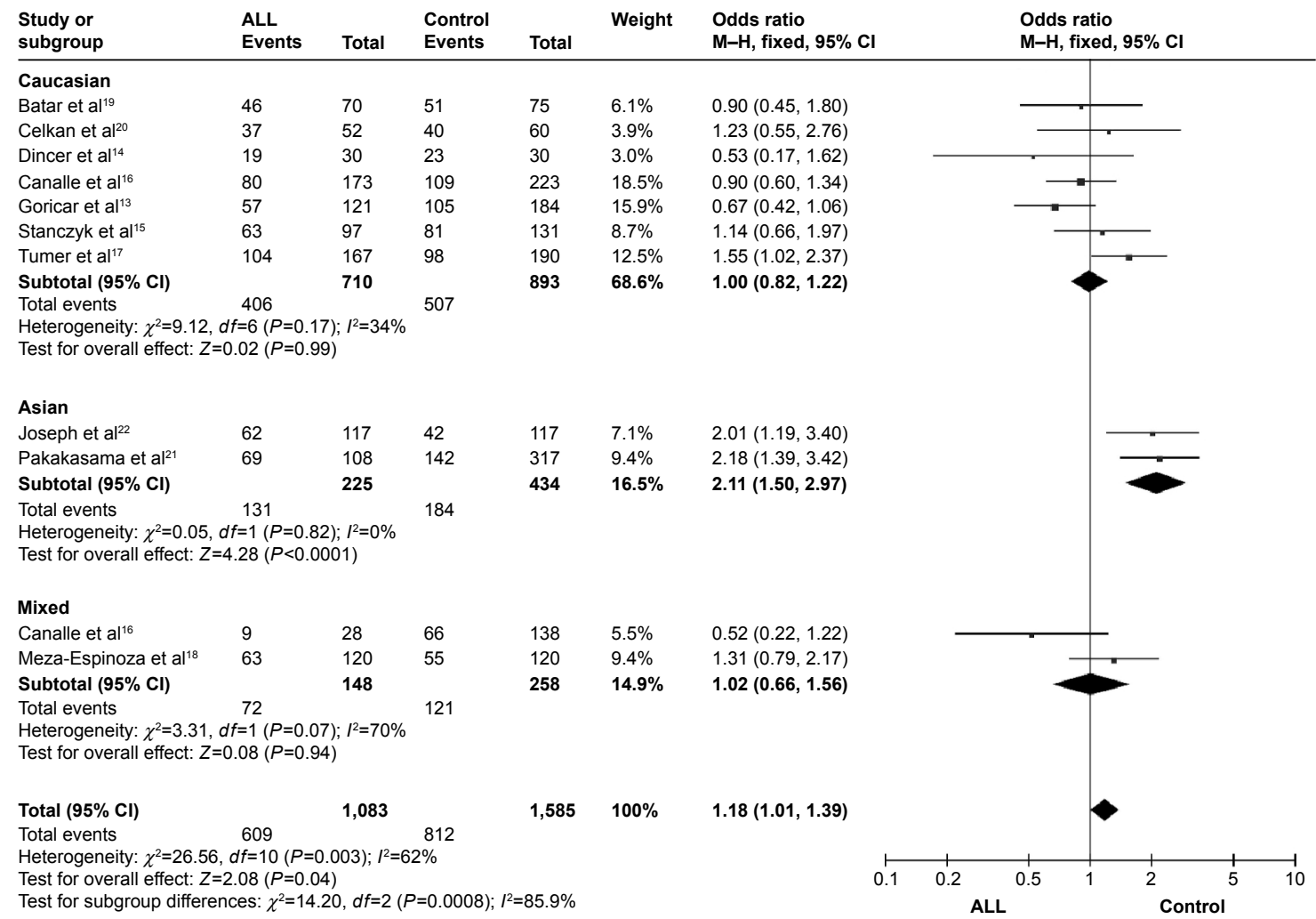

Figure 4 (Continued) 

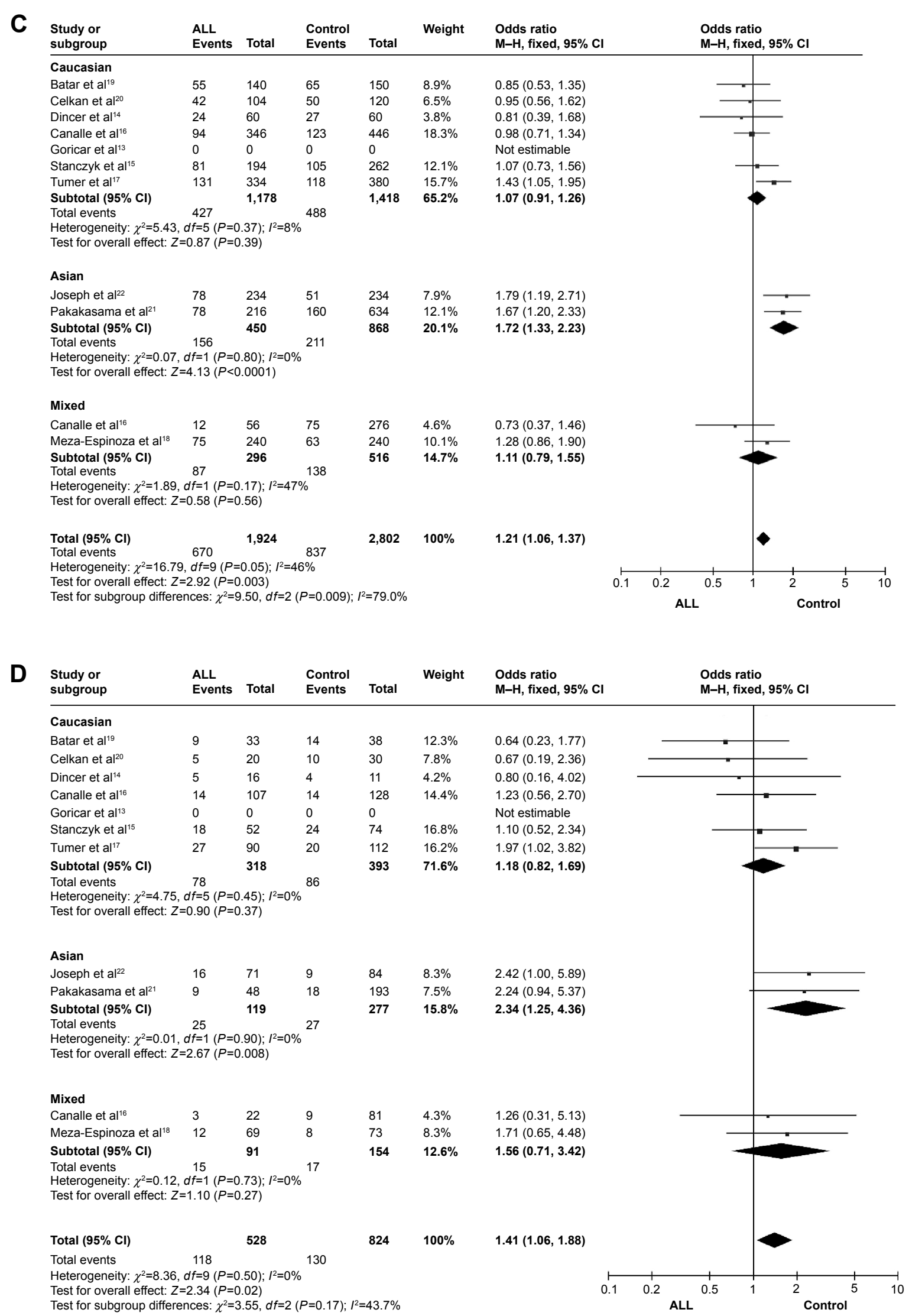

Figure 4 Meta-analysis of the association between XRCCI Arg399GIn SNP and ALL risk under the recessive model (A), the dominant model (B), the allele contrast model (C), and the homozygote contrast model (D).

Abbreviations: SNP, single-nucleotide polymorphism; ALL, acute lymphocytic leukemia; Cl, confidence interval; M-H, Mantel-Haenszel. 
as an outlier. Removal of that data from the analysis reduced the heterogeneity under all of the four contrast models.

Meta-analysis using fixed-effects model for the remaining two studies revealed no association between the SNP and risk of CLL under any contrast model (Table 2). Therefore, it indicates that the result about CLL is insensitive to the study of Duman et al. ${ }^{23}$

\section{Discussion}

A stratified analysis by leukemia type and ethnic group was conducted in this study in order to clarify the role of the XRCC1 Arg399Gln SNP in the development of four types of leukemia in different ethnic groups. Twenty-three studies were identified wherein research into the association between XRCC1 Arg399Gln SNP and different types of leukemia risk was conducted. Conclusions among these studies were not consistent, indicating an urgent need for the development of a systematic method for drawing more precise conclusions. Therefore, a common method of systematic review, a meta-analysis, was conducted in the present study to integrate these apparently contradictory findings with a view to yielding more accurate results. All of the included articles were of high quality and presented a rigorous scientific design, accurate data reporting, and clear results. The main conclusion was that the presence of the XRCC1 Arg399GIn SNP increased the risk of childhood ALL among Asians. No association was found between this SNP and either CML or CLL risk in Caucasians.

These conclusions suggest the existence of ethnic differences in childhood ALL, such that gene polymorphisms could result in ethnic-specific susceptibilities to leukemia. ${ }^{29}$ In addition, environmental factors such as birthplace and socioeconomic status may play critical roles in the genesis of leukemia. ${ }^{30}$ These factors might help to explain the reasons for the observed racial disparities.

The present findings suggest that the risk of childhood ALL is associated with DNA repair mechanisms. The XRCC1 Arg399Gln SNP may be useful as an important predictive factor, and ethnic background may have an impact on the role of this polymorphism on childhood ALL. This SNP may help to identify individuals at risk of developing ALL and represents an essential source of information for improvements in the treatment of ALL treatment.

The presence of heterogeneity between studies must be borne in mind when interpreting the results of a metaanalysis. ${ }^{31,32}$ Significant heterogeneity existed in the ALL group under the dominant model. After subgroup analyses by ethnicity, the heterogeneity decreased for Asian and Caucasian populations, but persisted in the mixed-race analysis, suggesting that ethnicity can account for the heterogeneity.

The meta-analysis for AML revealed no association between the SNP and risk of AML when random-effects modeling was performed. After excluding the study of Banescu et al, ${ }^{9}$ an outlier that caused high heterogeneity for the analysis of AML, the fixed-effects modeling, was used for the recessive model, and we found a changed result that the SNP was associated with AML risk in this genetic model, suggesting that the association between the SNP and risk of AML was sensitive to the data from the study of Banescu et al. ${ }^{9}$ In addition, the sample size for AML was small. Therefore, large-scale studies should be conducted in the future for more explicit and convincing results.

No association was found between the SNP and risk of either CML or CLL in Caucasians. It can be speculated that the development of chronic disease is greatly influenced by environmental factors, and that the influence of SNP might be weak. Accumulation of disadvantageous environmental factors might be required to develop chronic disease. Therefore, a study of environment-genetic interactions would be meaningful for this kind of chronic disease. Smoking and alcohol consumption are also important risk factors for leukemia. ${ }^{33}$ Although in the present study we endeavored to extract relevant information regarding smoking and drinking from the primary literature, insufficient data were obtained. Further investigations concerning the interactions between smoking, drinking, and gene variations with CLL and CML are required.

Only Caucasian subjects were included in the meta-analyses with respect to AML, CML, and CLL; therefore, the association between the SNP and risks of AML, CML, and CLL among other races needs to be determined in the future.

A meta-analysis similar to that presented herein was performed by Huang et al in 2014, ${ }^{1}$ who investigated the associations between XRCC1 Arg399Gln variations and leukemia susceptibility. However, one problem with the meta-analysis was that it was compromised by the inclusion of the study of Ozdemir et al, ${ }^{34}$ whose cohort comprised both ALL and Burkitt lymphoma patients who were not analyzed separately. Huang et $\mathrm{al}^{1}$ included the combined ALL and Burkitt lymphoma data of Ozdemir et $\mathrm{al}^{34}$ in their pooled ALL data, thus potentially yielding biased results. The study of Ozdemir et $\mathrm{al}^{34}$ was excluded from the present meta-analysis, which also included a greater number of studies than did the meta-analysis performed by Huang et al. ${ }^{1}$ In particular, the present meta-analysis of ALL included eleven articles, whereas Huang et $\mathrm{al}^{1}$ included only eight. 
The present statistically significant findings might, therefore, be more accurate than those of Huang et al. ${ }^{1}$

Two limitations of this meta-analysis should be considered when interpreting its findings. First, the number of eligible studies for the meta-analysis was small, and the results should thus be treated with caution. Further, this restricted the performance of the subgroup analyses. Second, only published studies were included in this meta-analysis. There is always a certain degree of publication bias, and nonsignificant or negative findings may be unpublished and thus underrepresented in the literature.

\section{Conclusion}

The findings of this meta-analysis indicate that the XRCC1 Arg399GIn SNP is associated with increased risk of childhood ALL in Asians. Large-scale studies should be conducted in the future to verify these results.

\section{Acknowledgments}

This work was supported by the Natural Science Foundation of Shaanxi Province (number 2015JM8415) and the Fundamental Research Funds for the Central Universities of China (number 08143047).

\section{Disclosure}

The authors report no conflicts of interest in this work.

\section{References}

1. Huang Y, Xie D, Tang N, et al. XRCC1 Arg399Gln variation and leukemia susceptibility: evidence from 2,647 cases and 5,518 controls. Tumour Biol. 2014;35(1):799-808.

2. Zhang H, Liu H, Jiang G. Genetic polymorphisms of XRCC1 and leukemia risk: a meta-analysis of 19 case-control studies. PLoS One. 2013; 8(11):e80687.

3. Seedhouse C, Bainton R, Lewis M, Harding A, Russell N, Das-Gupta E. The genotype distribution of the XRCC1 gene indicates a role for base excision repair in the development of therapy-related acute myeloblastic leukemia. Blood. 2002;100(10):3761-3766.

4. Berwick M, Vineis P. Markers of DNA repair and susceptibility to cancer in humans: an epidemiologic review. J Natl Cancer Inst. 2000;92(11): 874-897.

5. Ginsberg G, Angle K, Guyton K, Sonawane B. Polymorphism in the DNA repair enzyme XRCC1: utility of current database and implications for human health risk assessment. Mutat Res. 2011;727(1-2): $1-15$.

6. Higgins JP1, Thompson SG, Deeks JJ, Altman DG. Measuring inconsistency in meta-analyses. BMJ. 2003;327(7414):557-560.

7. DerSimonian R, Laird N. Meta-analysis in clinical trials. Control Clin Trials. 1986;7(3):177-188.

8. Egger M, Davey Smith G, Schneider M, Minder C. Bias in meta-analysis detected by a simple, graphical test. BMJ. 1997;315(7109):629-634.

9. Banescu C, Duicu C, Trifa AP, Dobreanu M. XRCC1 Arg194Trp and Arg399Gln polymorphisms are significantly associated with shorter survival in acute myeloid leukemia. Leuk Lymphoma. 2014;55(2): 365-370.
10. Sorour A, Ayad MW, Kassem H. The genotype distribution of the XRCC1, XRCC3, and XPD DNA repair genes and their role for the development of acute myeloblastic leukemia. Genet Test Mol Biomarkers. 2013;17(3):195-201.

11. Kim HN, Kim NY, Yu L, et al. Association of GSTT1 polymorphism with acute myeloid leukemia risk is dependent on smoking status. Leuk Lymphoma. 2012;53(4):681-687.

12. Ozcan A, Pehlivan M, Tomatir AG, et al. Polymorphisms of the DNA repair gene XPD (751) and XRCC1 (399) correlates with risk of hematological malignancies in Turkish population. Afr J Biotechnol. 2011; 10(44):8860-8870.

13. Goricar K, Erculj N, Faganel Kotnik B, et al. The association of folate pathway and DNA repair polymorphisms with susceptibility to childhood acute lymphoblastic leukemia. Gene. 2015;562(2):203-209.

14. Dincer Y, Yuksel S, Batar B, Guven M, Onaran I, Celkan T. DNA repair gene polymorphisms and their relation with DNA damage, DNA repair, and total antioxidant capacity in childhood acute lymphoblastic leukemia survivors. J Pediatr Hematol Oncol. 2015;37(5): 344-350.

15. Stanczyk M, Sliwinski T, Cuchra M, et al. The association of polymorphisms in DNA base excision repair genes XRCC1, OGG1 and MUTYH with the risk of childhood acute lymphoblastic leukemia. Mol Biol Rep. 2011;38(1):445-451.

16. Canalle R, Silveira VS, Scrideli CA, Queiroz RG, Lopes LF, Tone LG. Impact of thymidylate synthase promoter and DNA repair gene polymorphisms on susceptibility to childhood acute lymphoblastic leukemia. Leuk Lymphoma. 2011;52(6):1118-1126.

17. Tumer TB, Yilmaz D, Tanrikut C, Sahin G, Ulusoy G, Arinc E. DNA repair XRCC1 Arg399Gln polymorphism alone, and in combination with CYP2E1 polymorphisms significantly contribute to the risk of development of childhood acute lymphoblastic leukemia. Leuk Res. 2010; 34(10):1275-1281.

18. Meza-Espinoza JP, Peralta-Leal V, Gutierrez-Angulo M, et al. XRCC1 polymorphisms and haplotypes in Mexican patients with acute lymphoblastic leukemia. Genet Mol Res. 2009;8(4):1451-1458.

19. Batar B, Guven M, Baris S, Celkan T, Yildiz I. DNA repair gene XPD and XRCC1 polymorphisms and the risk of childhood acute lymphoblastic leukemia. Leuk Res. 2009;33(6):759-763.

20. Celkan T, Guven M, Batar B, Alhaj S. The difference between pre-B cell acute lymphoblastic leukemia and Burkitt lymphoma in relation to DNA damage repair gene polymorphisms in childhood. Leuk Lymphoma. 2008;49(8):1638-1640.

21. Pakakasama S, Sirirat T, Kanchanachumpol S, et al. Genetic polymorphisms and haplotypes of DNA repair genes in childhood acute lymphoblastic leukemia. Pediatr Blood Cancer. 2007;48(1):16-20.

22. Joseph T, Kusumakumary P, Chacko P, Abraham A, Pillai MR. DNA repair gene XRCC1 polymorphisms in childhood acute lymphoblastic leukemia. Cancer Lett. 2005;217(1):17-24.

23. Duman N, Aktan M, Ozturk S, et al. Investigation of Arg399Gln and Arg194Trp polymorphisms of the XRCC1 (x-ray cross-complementing group 1) gene and its correlation to sister chromatid exchange frequency in patients with chronic lymphocytic leukemia. Genet Test Mol Biomarkers. 2012;16(4):287-291.

24. Abramenko I, Bilous N, Chumak A, Kostin A, Martina Z, Dyagil I. DNA repair polymorphisms in B-cell chronic lymphocytic leukemia in sufferers of Chernobyl Nuclear Power Plant accident. J Radiat Res. 2012;53(3):497-503.

25. Ganster C, Neesen J, Zehetmayer S, et al. DNA repair polymorphisms associated with cytogenetic subgroups in B-cell chronic lymphocytic leukemia. Genes Chromosomes Cancer. 2009;48(9):760-767.

26. Banescu C, Trifa AP, Demian S, et al. Polymorphism of XRCC1, $\mathrm{XRCC} 3$, and XPD genes and risk of chronic myeloid leukemia. Biomed Res Int. 2014;2014:213790.

27. Annamaneni S, Gorre M, Kagita S, et al. Association of XRCC1 gene polymorphisms with chronic myeloid leukemia in the population of Andhra Pradesh, India. Hematology. 2013;18(3):163-168. 
28. Deligezer U, Akisik EE, Dalay N. Lack of association of XRCC1 codon 399Gln polymorphism with chronic myelogenous leukemia. Anticancer Res. 2007;27(4B):2453-2456.

29. Swinney RM, Beuten J, Collier AB 3rd, et al. Polymorphisms in CYP1A1 and ethnic-specific susceptibility to acute lymphoblastic leukemia in children. Cancer Epidemiol Biomarkers Prev. 2011;20(7): 1537-1542.

30. Bhatia S, Sather HN, Heerema NA, Trigg ME, Gaynon PS, Robison LL. Racial and ethnic differences in survival of children with acute lymphoblastic leukemia. Blood. 2002;100(6):1957-1964.

31. Zeng X, Zhang Y, Kwong JS, et al. The methodological quality assessment tools for preclinical and clinical studies, systematic review and meta-analysis, and clinical practice guideline: a systematic review. J Evid Based Med. 2015;8(1):2-10.
32. Zhang Y, Zhang J, Zeng L, et al. The $-2518 \mathrm{~A} / \mathrm{G}$ polymorphism in the MCP-1 gene and tuberculosis risk: a meta-analysis. PLoS One. 2012;7(7):e38918.

33. MacArthur AC, McBride ML, Spinelli JJ, Tamaro S, Gallagher RP, Theriault $\mathrm{G}$. Risk of childhood leukemia associated with parental smoking and alcohol consumption prior to conception and during pregnancy: the cross-Canada childhood leukemia study. Cancer Causes Control. 2008; 19(3):283-295.

34. Ozdemir N, Celkan T, Baris S, Batar B, Guven M. DNA repair gene XPD and XRCC1 polymorphisms and the risk of febrile neutropenia and mucositis in children with leukemia and lymphoma. Leuk Res. 2012;36(5):565-569.

\section{Publish your work in this journal}

OncoTargets and Therapy is an international, peer-reviewed, open access journal focusing on the pathological basis of all cancers, potential targets for therapy and treatment protocols employed to improve the management of cancer patients. The journal also focuses on the impact of management programs and new therapeutic agents and protocols on

\section{Dovepress}

patient perspectives such as quality of life, adherence and satisfaction. The manuscript management system is completely online and includes a very quick and fair peer-review system, which is all easy to use. Visit http://www.dovepress.com/testimonials.php to read real quotes from published authors.

Submit your manuscript here: http://www.dovepress.com/oncotargets-and-therapy-journal 